\title{
What you don't know can kill you
}

\author{
Geoffrey D. Barnes MD, MSc
}

Frankel Cardiovascular Center, Institute for Healthcare Policy and Innovation, University of Michigan Medical School, Ann Arbor, MI, USA

This is a commentary on Wendelboe et al [2017] doi: 10.1002/rth2.12051

Correspondence

Geoffrey D. Barnes, Frankel Cardiovascular Center, Institute for Healthcare Policy and Innovation, University of Michigan Medical School, Ann Arbor, MI, USA. Email: gbarnes@umich.edu

Atrial fibrillation (AF) is the most common cardiac arrhythmia worldwide, afflicting an estimated 33 million people. ${ }^{1}$ In the United States alone, AF-related annual cost estimates are as high as $\$ 26$ billion. While some patients experience bothersome symptoms, many remain completely asymptomatic. Most concerning is the severe consequence of untreated AF. Patients with AF are at an increased risk of stroke and systemic embolism, up to $15 \%$ per year. $^{2}$ In the United States, 800000 people suffer a stroke annually, and these strokes cause one in every 20 deaths. ${ }^{1}$ This converts to one stroke every 40 seconds and one stroke-related death every 4 minutes.

Despite these staggering statistics, there has long been anecdotal evidence that most of the at-risk population is unaware of AF or its association with stroke risk. In fact, prior studies have suggested that public screening of adults ages 65 and older would identify one new diagnosis of AF for every 170 adults screened. ${ }^{1}$ Additionally, inadequate understanding of how $\mathrm{AF}$ can cause stroke may contribute to the underutilization of, poor adherence to, and frequent early discontinuation of stroke preventative medications (eg, anticoagulants) among AF patients. ${ }^{3}$

To better understand the global awareness of AF, Wendelboe and colleagues conducted a global survey to quantify public awareness of $\mathrm{AF}$ in comparison to other thrombotic and non-thrombotic illnesses. ${ }^{4}$ Unsurprisingly, they found less than half (48\%) of those surveyed were aware of AF. This differed considerably from the high awareness of heart attacks (74\%), stroke (81\%), human immunodeficiency/acquired immunodeficiency syndrome (HIV/AIDS; 80\%), breast cancer (81\%), and prostate cancer (78\%). Additionally, they found a striking difference in awareness by country, with Uganda reporting the highest awareness (69\%) and Canada the lowest (25\%). While differences in the surveyed population and potential selection/responder bias may have contributed to part of the variation, a nearly three-fold difference is hard to ignore.

Perhaps the most important finding from this report is that only $36 \%$ of respondents were aware that AF can lead to stroke. With such prominent morbidity and mortality associated with stroke and the presence of a highly effective prevention method (anticoagulation), one would hope that the awareness of this connection was higher.

To help contextualize, the World Health Organization estimates 2.1 million global cases of HIV with one million yearly deaths. ${ }^{5}$ They also estimate annual deaths due to breast cancer around 500000 globally while the World Cancer Research Fund estimates 1.1 million global cases of prostate cancer annually. Each are incredibly important diseases worthy of robust public awareness campaigns. So too is AF, for which millions of people worldwide are at high risk of a potentially devastating stroke. Nonetheless, awareness of these conditions was strikingly higher than AF in this survey.

The role that advocacy organizations play in public awareness should not be underestimated. In fact, the World Health Organization has identified nine global public health days to raise awareness of HIV/AIDS, tobacco use, tuberculosis, and malaria. However, they do not have a day associated with AF, despite its sizeable prevalence and potentially devastating morbidity and mortality. Other well-funded and well-organized movements focused solely on HIV/AIDS and specific cancer types (eg, breast and prostate). It is no wonder that these conditions have gained widespread awareness across the globe. For the cardiovascular conditions, many major organizations (eg,the American Heart Association/American Stroke Association and the European Society of Cardiology) have focused on risk factor and lifestyle modification in their public health awareness campaigns. Additionally, these major cardiovascular organizations cover a wide range of diseases, from coronary artery disease and stroke to sudden cardiac death and atrial fibrillation. This diversity may limit the awareness of any specific disease, such as atrial fibrillation.

To help address a public knowledge gap regarding thrombosis, the International Society on Thrombosis and Haemostasis began World Thrombosis Day in $2014 .{ }^{6}$ This effort, in partnership with a number of other thrombosis-related organizations, focuses on public awareness of thrombotic disorders, primarily venous thromboembolism and AF.

This is an open access article under the terms of the Creative Commons Attribution-NonCommercial-NoDerivs License, which permits use and distribution in any medium, provided the original work is properly cited, the use is non-commercial and no modifications or adaptations are made.

(c) 2017 The Authors. Research and Practice in Thrombosis and Haemostasis published by Wiley Periodicals, Inc on behalf of International Society on Thrombosis and Haemostasis. 
To maximize the effect, the organizers have made two key strategic decisions.

First, World Thrombosis Day has chosen a multi-lateral approach to their awareness campaign. The have different events focused on public and professional awareness. They also use a variety of methods to spread their message, include social media, traditional media, and various in-person events. Other advocacy organizations, such as StopAfib.org also have a strong online presence to provide vital information to the public and to provide a community for patients and their family members. This type of approach is critical for reaching global audiences in a cost-conscious manner.

Second, World Thrombosis Day organizers have chosen to partner with local organizations to facilitate the activities of local organizations. This minimizes the burden on the primary organizing committee, engages more participants, and allows for better personalization based on the needs of individual communities and countries. It also allows for individual organizations to develop new and creative events that can be adapted and replicated broadly in subsequent years. For example, in October 2017, a World Thrombosis Day breakfast and walk occurred outside the Sydney International Convention Center while a Dog Parade was held in Mexico to promote walking as a thrombosis preventative (and treatment) strategy.

World Thrombosis Day has maintained a greater focus on venous thrombosis rather than AF awareness. This may reflect the interest of its originating organization, the International Society on Thrombosis and Haemostasis. It may also reflect a purposeful strategy to start with one clear focus and then slowly grow and expand into AF awareness over time. Either way, Wendelboe and colleagues highlight the desperate need for improved AF awareness worldwide.

These survey data will serve as an excellent baseline for measuring the impact of various public awareness campaigns, such as World Thrombosis Day. Yet, as important as measures of public awareness are, they are not the ultimate goal. Instead, our focus must be on increasing the use of preventative treatments as a means of reducing stroke. Improved rates of detecting $\mathrm{AF}$ and initiating stroke-preventative treatments are key metrics. The link between public awareness and these measures remains logical but without a strong evidence base. Nonetheless, the efforts are a critical component of achieving the ultimate goal (stroke reduction) and worthy of global support.

The National Heart, Lung, and Blood Institute at the United States National Institutes of Health recently published a clinical practice guideline on implementation strategies for clinical practice guidelines. ${ }^{7}$ That report outlines a multi-level framework involving a policy level, clinical institution levels, provider level, and patient level. Within the patient level is an assessment of knowledge, which is directly impacted by health awareness campaigns. However, equally important is the provider level use of screening and diagnosis along with treatment and procedural decision making. The framework also highlights the important role of institutions to support educational outreach and clinical decision support. The survey data from Wendelboe and colleagues would suggest that educational outreach must extend beyond provider education to also include educating the public. ${ }^{7}$

To achieve the goal of reduced AF-related stroke burden we must target preventative and therapeutic strategies in addition to awareness. For example, we must encourage efforts to reduce the development of AF, such as increasing physical activity, weight loss, and treatment of comorbid illnesses (patient and policy levels). The public lexicon should include "AFib" just as easily as it does "heart attack," "stroke," and "cancer." We also need to address issues surrounding medication prescribing, access, adherence, and persistence (patient, provider, institutional, and policy levels). These will require multifaceted efforts to develop, test, implement, and promote care programs. Public health and governmental organizations, pharmaceutical companies, insurers and other payers, professional societies and advocacy organizations, healthcare providers, and patients all must work together to increase the use of stroke-preventative treatment strategies among patients with atrial fibrillation.

Together, we can make important strides in both public awareness of $\mathrm{AF}$ and the use of stroke-preventative measures. With regards to public awareness, we now have our starting point. How we reach our ultimate goal remains an exciting and uncharted journey.

\section{ACKNOWLEDGMENTS}

I wish to thank Shashank Sinha, MD, MSc, for his critical review of this manuscript.

\section{RELATIONSHIP DISCLOSURES}

Dr. Barnes reports grants and personal fees from Janssen, grants and personal fees from Pfizer/Bristol-Myers Squibb, personal fees from Portola, grants from Blue Cross Blue Shield of Michigan, and grants from National Heart, Lung, and Blood Institute.

\section{ORCID}

Geoffrey D. Barnes iD http://orcid.org/0000-0002-6532-8440

\section{REFERENCES}

1. Writing Group M, Mozaffarian D, Benjamin EJ, et al. Heart Disease and Stroke Statistics-2016 update: a Report From the American Heart Association. Circulation. 2016;133:e38-360.

2. Lip GY, Nieuwlaat R, Pisters R, Lane DA, Crijns HJ. Refining clinical risk stratification for predicting stroke and thromboembolism in atrial fibrillation using a novel risk factor-based approach: the euro heart survey on atrial fibrillation. Chest. 2010;137:263-72.

3. Barnes GD, Kaatz S, Lopez A, et al. Discontinuation of warfarin therapy for patients with atrial fibrillation: The Michigan Anticoagulation Quality Improvement Initiative Experience. JAMA Cardiol. 2017;2: 341-3.

4. Wendelboe AM, Raskob GE, Angchaisuksiri P, et al. Global public awareness about atrial fibrillation. Res Pract Thromb Haemost. 2018;2:49-57. 
5. World Health Organization: Global Health Observatory Data 2017 [cited 2017 Oct 7]. Available from http://www.who.int/gho/en/.

6. World Thrombosis Day [cited 2017 Oct 7]. Available from http://www. worldthrombosisday.org/.

7. Chan WV, Pearson TA, Bennett GC, et al. ACC/AHA Special Report: clinical practice guideline implementation strategies: a summary of systematic reviews by the NHLBI Implementation Science Work Group: a Report of the American College of Cardiology/American Heart Association Task Force on Clinical Practice Guidelines. J Am Coll Cardiol. 2017;69:1076-92.

How to cite this article: Barnes GD. What you don't know can kill you. Res Pract Thromb Haemost. 2018;2:8-10. https://doi. org/10.1002/rth2.12056 